\title{
Non destructive testing of medium and high voltage cables with a transportable radiography system
}

\author{
J. G. Fantidis ${ }^{1, *}$, C. Potolias ${ }^{2}$, D. V. Bandekas ${ }^{2}$ and N. Vordos ${ }^{2}$ \\ ${ }^{1}$ Laboratory of Nuclear Technology, School of Engineering, “Democritus” University of Thrace, Xanthi, Greece. \\ ${ }^{2}$ Dept. of Electrical Engineering, Kavala Institute of Technology, Greece.
}

Received 8 January 2010; Revised 25 February 2010; Accepted 30 March 2010

\begin{abstract}
A power cable is the most important part in a power transmission system. The cables must be total quality dedicated and certified for development, manufacturing and installation, however are exposed to a corrosive environment. The purpose of this paper is to show that the fast neutron radiography with a transportable system is a solution to find defects in the cables and reduce the cost of inspection. The design, regarding the materials considered, was compatible with the European Union Directive on "Restriction of Hazardous Substances" (RoHS) 2002/95/EC, hence excluding the use of cadmium and lead. Wide width values for the collimator ratio were calculated. With suitable collimator design it was possibly to optimize the neutron radiography parameters. Finally the shielding design was examined closely. The proposed system has been simulated using the MCNPX code.
\end{abstract}

Keywords: Medium-high voltage cable, Fast neutron radiography, MCNPX, DD neutron generator.

\section{Introduction}

The electric utility industry has used power cables for transmission and distribution of electric power for years. The power cables currently in use are composed of polyolefins which have been the industry standard since the early sixties. High and medium voltage cables dominate the cable market segment. In a typical high and medium voltage cable (Fig. 1), copper or aluminium wires are used as the conductors. These conductors are covered with an extruded polymeric stress-control layer made of semi-conductive compounds. All high and medium voltage power cables have an insulation layer. If a conductor is energized at line voltage or potential, it is obviously necessary to isolate the conductor or line electrode from any ground or ground electrode. Insulating materials have to meet very demanding requirements just like high dielectric strength, low dielectric constant, low power factor, moisture resistance, high resistance to heat and high resistance to corona damage. The copper neutral wires are wound around the insulation shield and are usually covered with a thermoplastic polyethylene jacket for mechanical protection from the external environment and to reduce moisture intrusion into the cable, all of which can cause the premature cable failure [1].

Cables consist of three major components: conductors, insulation and protective jacket. The composition of individual cables varies according to the application. The construction and mate- rial are determined by three main factors: rated voltage, conductor cross section and environmental conditions. Suitable materials must be used to achieve a good quality cable. Today sophisticated intermediate tests during manufacture of all material and of the completed cable core assure compliance with highest quality standards as mentioned in IEC 60502 Part 2 [2, 3]. However ca-

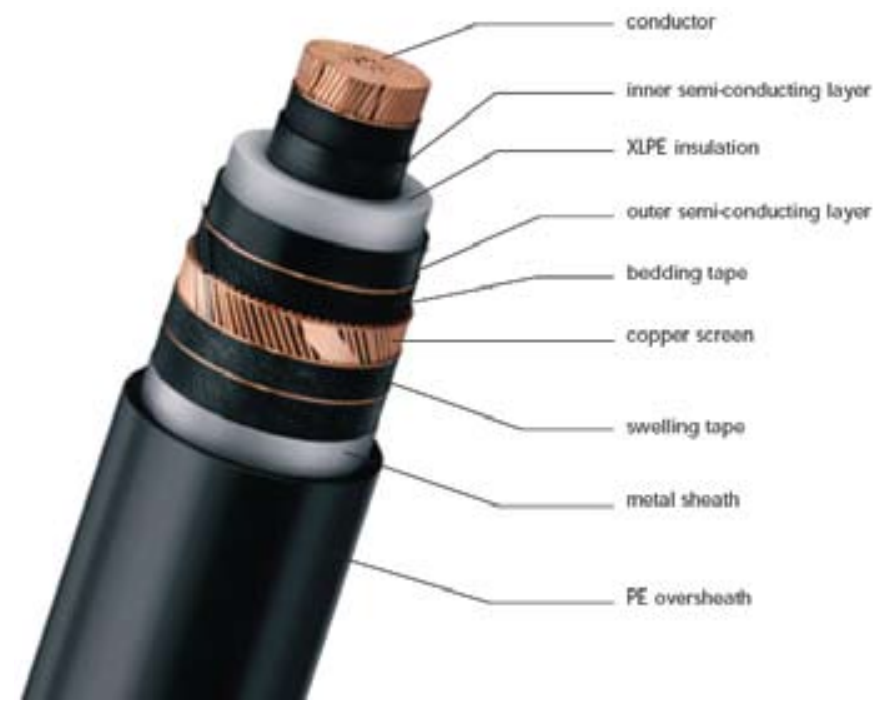

Figure 1. A typical medium voltage cable 
bles must be inspected and after manufacture, and often during their service life, to guarantee that their condition is suitable for their purpose. Non-destructive Testing (NDT) plays a vital role in assuring that cables perform their function in a reliable way. NDT can be defined as "inspections, tests, or evaluations which may be applied to a structure or component to determine its integrity, composition, electrical or thermal properties, or dimensions without causing a change in any of these characteristics" [4].

Radiography is the image records for internal structures of the object with X-rays, gamma rays, or neutrons passing through object to the film or detector. The image records used to be film, and now are digital image. Radiography today is one of the most important, most versatile, of all the NDT methods used by modern industry [5]. Neutron radiography (NR) is a powerful non-destructive testing technique frequently used, either on its own or as complementary to X-ray radiography, for the analysis of objects.

Imaging with neutrons works identically to the medical x-ray imaging. A sample is irradiated by a well defined beam with a small divergence and the transmitted neutron flux is measured by a position sensitive neutron detector. The grey levels in the radiography image correspond to the transmitted intensity of the object. Since neutrons are free particles, do not interact with the electrical charge of the electrons within atoms, but with the atomic nuclei. The thermal neutron mass attenuation coefficient shows no correlation with the atomic number. This unique feature makes a neutron capable of detecting elements or even isotopes that cannot be seen by X-ray. In contrast to $\mathrm{X}$-rays, thermal neutrons are particularly attenuated by certain light materials $(\mathrm{H}, \mathrm{Li}, \mathrm{B}, \mathrm{C}$, ) whereas they penetrate easily through heavy elements $(\mathrm{Pb}, \mathrm{Bi}, \mathrm{U}, \mathrm{W})$ [6]. The technique is widely used in security applications, in automotive and aerospace applications, engineering studies and industry in order to determine structural defects, geology, medicine and biological research, [7-17]. In the high voltage cables there are simultaneously more than one light material (usually XLPE and PVC) which is practical impossible to distinct with the traditional X-ray systems [18-20].

Most worldwide work in NR has been done using thermal neutrons. This occurs as a result of the availability of high-intensity thermal neutron beams from nuclear research reactors and neutron sources and because of the large variations among the neutron scattering and absorption cross sections of different elements. However, their use is basically limited to relatively thin objects. Fast neutrons are highly penetrating and are, therefore, attractive when bulky objects have to be investigated. For example, cracks in thick $(10-30 \mathrm{~cm})$ objects can be identified using fast neutrons. Fast neutron radiography (FNR) with neutron energies greater than $1 \mathrm{MeV}$, could open up a new range of possibilities for non destructive inspections. In order to produce good quality radiographies from an intense source of fast neutrons still requires some ingenuity, as fast neutrons are not very easy to collimate. This is due to the lack of sufficiently strong absorbers for fast neutrons which are needed to construct apertures or collimator walls. Nevertheless, fast neutron imaging systems can be effectively built and used. In perspective with thermal neutron there is little variation in attenuation among the elements when neutron energies are in the $\mathrm{MeV}$ range [21-22]. However, the number of nuclei per unit volume does vary from element to element. Last but not least a transportable fast neutron radiography unit is not easy to set up because high energy neutrons require bulky shielding.

\section{Materials and methods}

\subsection{The neutron generator and the radiography unit}

In this work, a transportable unit for FNR, incorporating a Deuterium-Deuterium (DD) neutron generator, has been simulated using the MCNPX Monte Carlo code [23]. A transportable unit would offer the prospect to enlarge the range of applications of FNR. The aim of the work is to design a transportable unit, which is suitable to investigate medium and high voltage cables, ensuring simultaneously sufficient occupational radiation protection measures for the personnel in the neighbourhood of the unit. The proposed unit is designed according to article 4 of the RoHS Directive 2002/95/EC, regarding the choice of materials. Hence, lead, mercury, cadmium, hexavalent chromium, polybrominated biphenyls and polybrominated diphenyl ethers have been excluded from the design [24].

A variety of neutron beams are available for fast NR, with their choice been a compromise between beam intensity and transportability for in-situ testing. Nuclear reactors and acceleratordriven neutron sources provide high intensity neutron beams at the expense of transportability. On the contrary, a range of commercially available isotopic neutron sources, such as ${ }^{241} \mathrm{Am} / \mathrm{Be}$ and ${ }^{252} \mathrm{Cf}$, can be easily incorporated in transportable radiography units, at the expense of beam intensity and require shielding enduringly.

The DD (Deuterium-Deuterium) neutron generators seem to be a smart choice because they produce neutrons, with energy approximately equal to $2.5 \mathrm{MeV}$, suitable for fast NR. Furthermore, they offer an on/of switching of the emitted neutrons. They have a compact size, logical cost and a relatively high neutron flux. In this work, a compact neutron generator from NSD-Fusion GmbH (model NSD-NG-1.25e9-DD-P) was simulated [25]. The NSD neutron generator has exceptionally long life due to its plasma target over a solid target (according to the company probably up to 40000 hours) without needing service. It is powerful portable neutron generator and capable of delivering $1.25 \times 10^{9} \mathrm{n} \mathrm{s}^{-1}$. The neutron generator has cylindrical shape with overall dimensions $130 \mathrm{~cm}$ length and emission unit diameter $13.4 \mathrm{~cm}$.

The system is designed in the form of one or more cylinders closed at one edge while the other edge (side) is open (Fig. 2). The walls of the cylinders are made of shielding materials surround the neutron generator. Next to the open edge is the imaging neutron collimator of variable length. The cable under examination is placed, together with the image recording apparatus, in front of the collimator inlet next to the image plane, D.

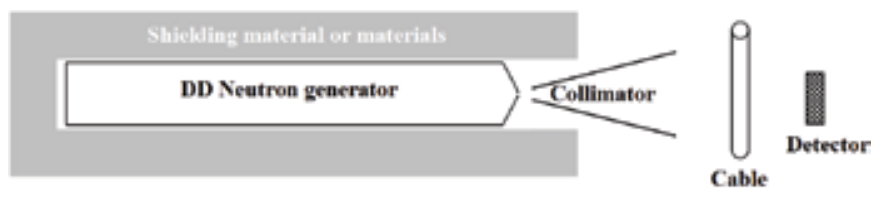

Figure 2. Side view of the geometric configuration of the irradiating system (not in scale)

\subsection{Fast neutron collimator}

The quality of fast NR imaging is determined by the collimator ratio $(L / D)$ where $\mathrm{L}$ is the collimator length and $\mathrm{D}$ is the colli- 
mator aperture diameter. The considerable penetration of fast neutrons inside an object results to a geometric unsharpness $u_{g}$ given by the equation [26]

$u_{g}=\frac{D t}{L_{D}-t}$

where $L_{D}$ is the distance from the aperture to the image plane and $t$ is the image surface to object distance. The imaging quality of a fast neutron radiography facility would be further characterized by the beam quality profile, described by the number of uncollided neutrons which reach the detector position within the neutron beam.

The lack of sufficiently strong absorbers for fast neutrons results in neutrons emerging from the walls of a collimator. Some of these re-merging neutrons can scatter towards the area of exposure projected by the collimator on the object. Scattering from the collimator walls would also produce slower neutrons that can change the energy of the extracted neutron beam. These epithermal or thermal neutrons downgrade the quality of the extracted beam, which should have the same energy distribution as that of the source (uncollided neutrons). Unfortunately the neutron moderating materials which are used to thermalise neutrons they are also good scattering materials. Scattering can get worse significantly the quality of the beam profile. Therefore, metals are found to be more suitable as collimator material for fast neutrons. Then, neutrons would be scattered away from the interior walls of a collimator following very few collisions, with an insignificant loss in energy. The low cost of iron (Fe) compare with tungsten, and the difficulties associated with tungsten fabrication, make the iron the best solution [27].

The fast neutron collimator, considered in this work, comprises two parts (Fig. 3). The first which is a conic convergent one, with a length of $14 \mathrm{~cm}$ and radii of $1.5 \mathrm{~cm}$ and $0.5 \mathrm{~cm}$, inside a HD-PE cylinder with a radius of $8 \mathrm{~cm}$. The second collimator which is a divergent one, with an inlet aperture of $1 \mathrm{~cm}$, a variable length and walls made of 4 layers of different materials. These materials from the inside outwards are: $7 \mathrm{~cm}$ thick iron $(\mathrm{Fe}), 0.5 \mathrm{~cm}$ thick gadolinium $(\mathrm{Gd}), 2 \mathrm{~cm}$ thick $\mathrm{PE}-\mathrm{B}$ and $1.5 \mathrm{~cm} \mathrm{Bi}$.
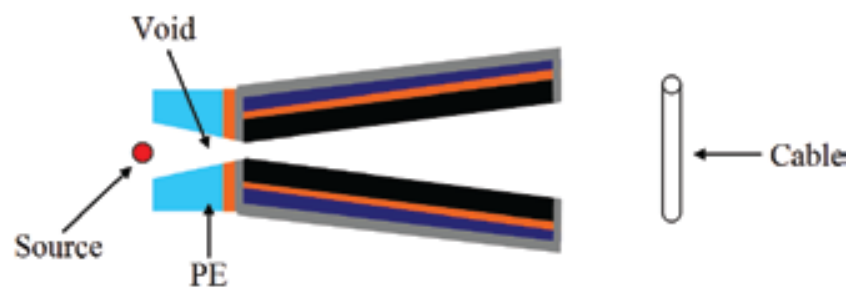

collimator

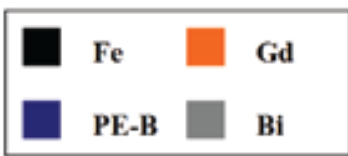

Figure 3. The aperture geometry and the collimator for fast neutron radiography (not in scale)

This first collimator is able to absorb some scattered neutrons and eliminate their energy and population towards the second col- limator, therefore improving the quality of the beam profile. The aperture, placed between the two parts, is a combination of two materials: a $0.3 \mathrm{~cm}$ and $1 \mathrm{~cm}$-thick layers of $\mathrm{Gd}$ and $\mathrm{Bi}$ used to absorb thermal neutrons while preventing the gamma rays generated within the other materials to reach the second collimator. In all circumstances there is a $2 \mathrm{~cm}$ thick layer of $\mathrm{Bi}$ at the latter stage of collimator, to provide shielding against any gamma-rays that may have been produced within the collimator's material by neutron activation. The object to detector distance controls the recording of scattered neutrons. Recording scattered neutrons leads to decreased imaging contrast. When the object to detector distance increase the scattered neutron intensity decreases by $1 / t^{2}$ but the source neutron intensity in the detector plane decreases because at the same time increase the distance from aperture to the image plane length of primary collimator.

In this paper two different cables investigated. The first is a medium voltage cable from Nexans Corporation (type $2 \mathrm{XSY} / 1 \times$ $120 \mathrm{RM} / 16$ ) for $8.7 / 15 \mathrm{kV}$. The electric and insulation characteristics of the cables are given in Table 1. Figure 4 shows a schematic side view of this cable. In Fig. 5, the geometric configuration of a typical high voltage cable from the same company (type $2 \mathrm{X}(\mathrm{F})$ $\mathrm{K} 2 \mathrm{Y}, 220 / 380 \mathrm{kV}$ ) is shown. In all simulation cables consist of the three major components (conductors, insulation and protective jacket) [28-29].

Table 1. The electric and insulation characteristics of the cables.

\begin{tabular}{l|c|c}
\hline & 2XSY & 2XS(FL)2Y \\
\hline Rated Voltage Uo/U (Um) & $8.7 / 15 \mathrm{kV}$ & $220 / 380 \mathrm{kV}$ \\
Cross section [mm $\left.{ }^{2}\right]$ & 120 & 1000 \\
Screen section $\left[\mathrm{mm}^{2}\right]$ & 16 & 50 \\
Nom. Outer diam. [mm] & 32 & 115 \\
Thickness of insulation [mm] & 4.5 & 26 \\
Approx. Weight [kg/m] & 1.95 & 27 \\
Perm. current rating buried $20^{\circ} \mathrm{C}-$ & 366 & 720 \\
trefoil formation [A] & 413 & 797 \\
Current rating in air $30^{\circ} \mathrm{C}-$ trefoil $[\mathrm{A}]$ & & \\
\hline
\end{tabular}
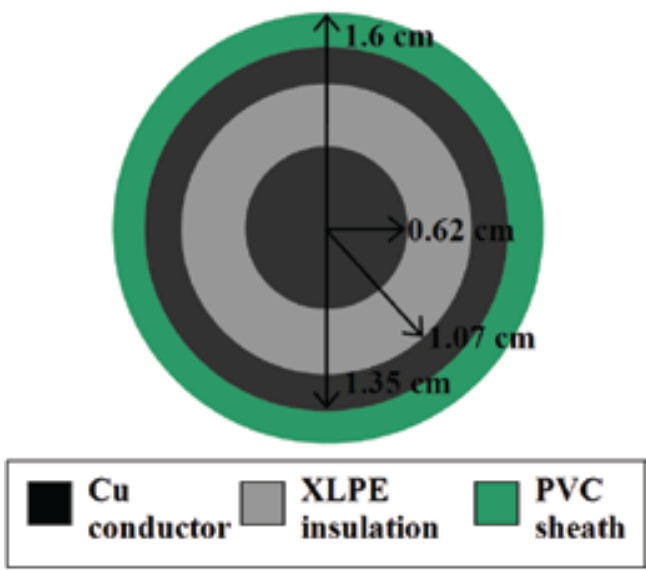

Figure 4. The major components of a typical medium voltage cable

Finally, the necessary shielding for radiation protection purposes was optimized using the MCNPX 2.5.0 code. A range of materials were considered (Table 2), which would provide effective shielding while still rendering the unit transportable. Borated poly- 
ethylene (PE-B) is polyethylene with $5 \%$ boron and is widely used in neutron shielding applications because of its good nuclear and physical characteristics. In comparison with PE-B two advanced neutron shielding materials were studied, zirconium borohydride $\mathrm{Zr}\left(\mathrm{BH}_{4}\right)_{4}$ and magnesium borohydride $\mathrm{Mg}\left(\mathrm{BH}_{4}\right)_{2}$ [30-31]. Bismuth (Bi) is an excellent material for gamma-ray filtering. The shielding material placed outside the DD neutron generator, comprise different materials with variable thicknesses, bearing in mind the transportability of the unit. The Dose Equivalent Rates (DER) was calculated at positions which are closest to the source, where the dose has the maximum value.

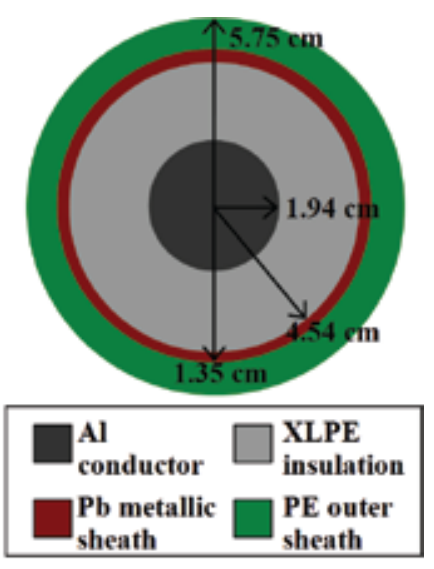

Figure 5. The major components of a typical high voltage cable

Table 2. Neutron shielding materials and their characteristics.

\begin{tabular}{l|c|c|c}
\hline \multicolumn{1}{c|}{ Material } & $\begin{array}{c}\text { Density } \\
\left(\mathbf{g ~ c m}^{-3}\right)\end{array}$ & $\begin{array}{c}\text { Mass } \\
\text { number }\end{array}$ & $\begin{array}{c}\mathbf{H ~ d e n s i t y ~} \\
\left(\mathbf{1 0}^{22} \mathbf{c m}^{-3}\right)\end{array}$ \\
\hline Borated polyethylene $(5 \% \mathrm{~B})$ & 0.94 & 17.04 & 6.6 \\
Zirconium borohydride $\left(\mathrm{Zr}\left(\mathrm{BH}_{4}\right)_{4}\right)$ & 1.18 & 150.60 & 7.2 \\
Magnesium borohydride $\left(\mathrm{Mg}\left(\mathrm{BH}_{4}\right)_{2}\right)$ & 1.48 & 53.99 & 13.2 \\
\hline
\end{tabular}

\section{Results and discussion}

The shielding was designed taking into consideration: the RoHS directive, the weight and dimensions which would render the unit transportable and the annual occupational dose limit of 0.5 $\mathrm{Sv}$ (or $25 \mu \mathrm{Sv} \bullet \mathrm{hr}^{-1}$ ). The total dose was calculated with the MCNPX Monte Carlo code, using the F2, and Fm2 tallies combined with the DE and DF cards. The F tallies describe the neutron flux within a surface, while the two cards convert the absorbed dose to the Dose Equivalent Rate (DER). Calculations were performed for a total number of histories per starting neutron (NPS) of $10^{8}$ yielding accuracy in the calculations of $<1 \%$. Figure 6 shows the resulting dose rates at the surface of the transportable unit for different thicknesses of the shielding materials. The total dose comprises the neutrons and the photons, the latter been from the interaction of the neutrons and the moderator material. The total DER estimates, obtained for combinations of different layers of the materials shown in Table 3, are given in Table 2. PE-B is desirable for use in any location requiring neutron shielding; however $\mathrm{Zr}\left(\mathrm{BH}_{4}\right)_{4}$, and specially the $\mathrm{Mg}\left(\mathrm{BH}_{4}\right)_{2}$ according to the
Table 3 show superior shielding capability than the conventional materials such as PE-B.

Table 3. Estimates of the dose rate and weight for different shielding configurations.

\begin{tabular}{l|c|c|c|c|c|c|c|c}
\hline & \multicolumn{6}{|c|}{ Shielding materials thickness } & $\begin{array}{c}\text { Weight } \\
\text { (kg) }\end{array}$ & \multicolumn{3}{|c}{ Dose rate } \\
\hline & $\mathbf{P E - B}$ & $\mathbf{Z r}\left(\mathbf{B H}_{4}\right)_{4}$ & $\mathbf{M g}\left(\mathbf{B H}_{4}\right)_{2}$ & $\mathbf{B i}$ & & Neutrons & Photons & Total \\
\hline Layer 1 & & & 50 & & 2985 & 8.85 & 13.99 & 22.84 \\
Layer 1 & 45 & & & & 3315 & 13.52 & 10.87 & 24.38 \\
Layer 2 & & & & 1 & & & & \\
Layer 1 & & 31 & & & 2060 & 7.05 & 17.07 & 24.12 \\
Layer 2 & & & & 1 & & & &
\end{tabular}

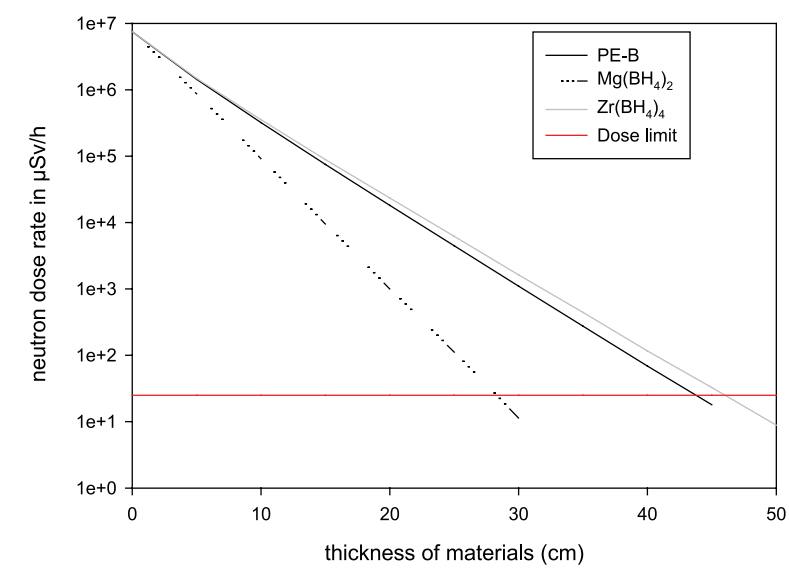

Figure 6. Calculated neutron dose rates for as a function of the thickness of different shielding materials

The suggested system comprises a collimator with a variable collimator length $(L=50-150 \mathrm{~cm})$, diameter of its aperture next to the image plane $\left(D_{0}=14-22 \mathrm{~cm}\right)$ and divergence angle $(\theta)$ of the beam $\left(\theta=4.2-8.0^{\circ}\right)$, while the inlet aperture $(D)$ of the collimator is $1 \mathrm{~cm}$. Fast neutron fluxes $\left(f_{F}\right)$ were calculated with the aid of MCNPX code using the F2 tally with NPS $=3 \times 10^{7}$ neutrons yielding an accuracy $<0.5 \%$ (Table 4 ). The object - detector distance $(t)$ was considered to be 25 . The distance between the end of the collimator and the detector was $2 t$. The collimator weight varies between $230-680 \mathrm{~kg}$.

Table 4. The fast NR calculated parameters using the proposal system.

\begin{tabular}{c|c|c|c|c|c|c}
\hline $\begin{array}{c}\mathbf{L} \\
(\mathbf{c m})\end{array}$ & $\mathbf{L} / \mathbf{D}$ & $\begin{array}{c}\mathbf{D}_{\mathbf{0}} \\
(\mathbf{c m})\end{array}$ & $\begin{array}{c}\boldsymbol{\theta} \\
(\boldsymbol{})\end{array}$ & $\begin{array}{c}\mathbf{U}_{\mathbf{g}} \\
(\mathbf{c m})\end{array}$ & $\begin{array}{c}\mathbf{f F} \\
\left(\mathbf{n ~ c m}^{-2} \mathbf{s}^{-1}\right)\end{array}$ & $\begin{array}{c}\text { Uncollided } \mathbf{f}_{\mathbf{F}} \\
(\mathbf{\%})\end{array}$ \\
\hline 50 & 50 & 14 & 7.96 & $5.00 \mathrm{E}-1$ & $1.21 \mathrm{E}+4$ & $96.48 \%$ \\
75 & 75 & 16 & 6.08 & $3.33 \mathrm{E}-1$ & $7.36 \mathrm{E}+3$ & $98.05 \%$ \\
100 & 100 & 18 & 5.14 & $2.50 \mathrm{E}-1$ & $4.97 \mathrm{E}+3$ & $98.67 \%$ \\
125 & 125 & 20 & 4.57 & $2.00 \mathrm{E}-1$ & $3.59 \mathrm{E}+3$ & $99.02 \%$ \\
150 & 150 & 22 & 4.19 & $1.67 \mathrm{E}-1$ & $2.71 \mathrm{E}+3$ & $99.22 \%$ \\
\hline
\end{tabular}

The calculated parameters for the fast neutron radiography system are shown in Table 4. The uncollided fast neutron flux, 
which illustrates the beam quality, ranges between 96.5 to $99.2 \%$. The fast neutron flux at the field of view at the object was uniform to within $1 \%$ and varies from $2.7 \times 10^{3}$ up to $1.2 \times 10^{4} \mathrm{n} \mathrm{cm}^{-2} \mathrm{~s}^{-1}$. According to Mikerov and Waschkowski the detection of defects $0.1 \mathrm{~cm}$ in size, would require an exposure of about $1.5 \times 10^{7}$ fast neutron $\mathrm{cm}^{-2}$ [32], with the exposure time being proportional to the fast neutron flux. In the case of $L / D=50$, the exposure time is $20.7 \mathrm{~min}$. Higher $L / D$ values would require higher exposure time: in the cases of $L / D=100$ and 150 , exposure times are 50.3 and $92.3 \mathrm{~min}$ respectively.

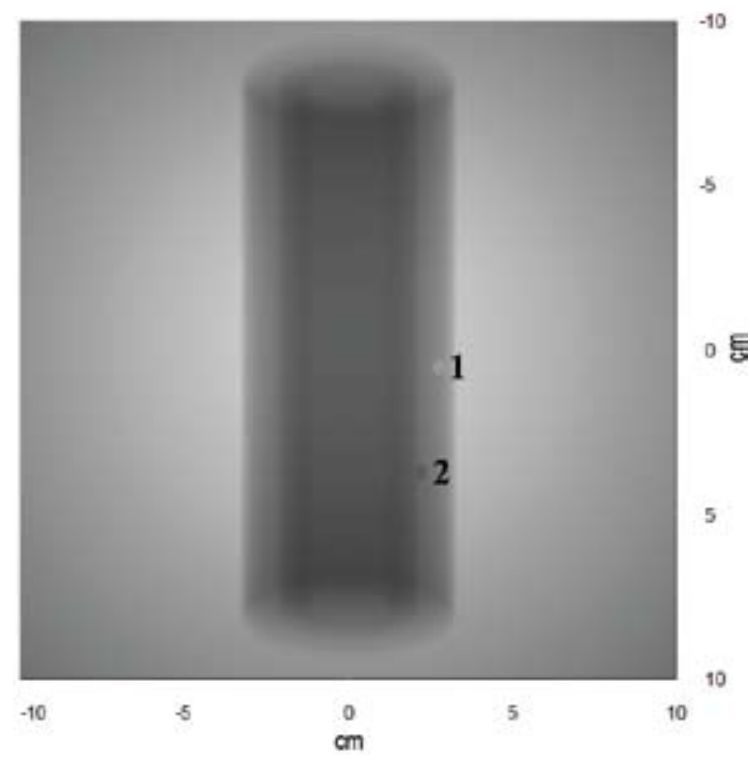

Figure 7. Fast neutron radiography of a typical medium voltage cable

In Fig. 7 a radiographic image of a typical medium voltage cable is presented. In the cable there are two defects, a small void sphere (1) with radius $0.75 \mathrm{~cm}$ and a second small sphere (2) with the same radius which contains water. Correspondingly in Fig. 8 a typical high voltage cable contain a small void sphere (3) with radius $0.75 \mathrm{~cm}$. Both figures show that using fast neutrons from a DD neutron generator, the proposal transportable system is capable to detect defects in a cable. This methods are better than the using of X-rays in the inspection of medium and high voltage cables [18-20] because fast neutrons not only have good penetration in metals $(\mathrm{Cu}$ or $\mathrm{Al})$ and heavy materials $(\mathrm{Pb})$ but also there is variation in a light materials (XLPE, PVC).

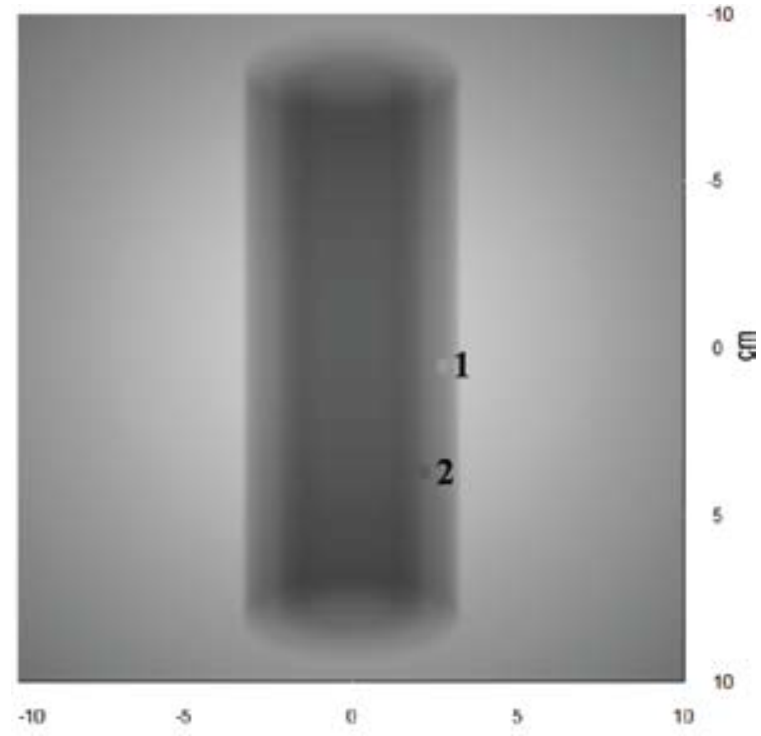

Figure 8. Fast neutron radiography of a typical high voltage cable

\section{Conclusion}

A transportable system using a DD compact neutron generator has been simulated, for radiography purposes, using the MCNPX Monte Carlo code. All the materials considered were chosen according to the EU Directive 2002/95/EC, excluding lead and cadmium. The system was designed under the constraint that the $D E R$ should remain below the annual occupational dose limit. The design was optimized with respect to neutron shielding and collimation. Advanced neutron shielding materials, has been shown in this work to be the best candidate to replace PE-B when maximum portability is required. According to the results obtained, the proposed system has dimensions which render it suitable for transportation with a light stake truck. In relation to the results obtained, the simulated facility has a wide range of values for the parameters characterising the fast neutron radiographies resulting in radiographs of variable quality. Radiography with fast neutrons made possible in extracting internal defects of cables which is not possible through conventional methods which use X-ray imaging technique.

\section{References}

1. www.nexans.de

2. www.abb.com/cables

3. http://www.iec.ch/ (as described in IEC 60502 Part 2)

4. FAA, Advisory Circular 65-31A, Training, Qualification and Certification of Non-destructive Inspection Personne, 2003.

5. Quinn R. A., Sigl C. C., Radiography in Modern Industry, fourth edition, Rochester, New York Eastman Kodak Company, 2001.

6. Lehmann E. and Kardjilov N., in: J. Banhart (Ed.), Advanced Tomographic Methods in Materials Research and Engineering, Oxford University Press, Oxford, 2008.

7. Bennett L. G. I., Chalovich T. R., Lewis W. J., IEEE Trans. on Nucl. Scien. 52 (2005) pp. 334-337.
8. Eberhardt J. E., Rainey S., Stevens R. J., Sowerby B. D., Tickner J. R., Appl. Radiat. Isot. 63(2) (2005) pp.179-188.

9. Liu Y., Sowerby B. D., Tickner J. R., Appl. Radiat. Isot. 66 (2008) 463.

10. Fiori F., Giunta G., Hilger A., Kardjilov N., Rustichelli F., Physica B 1206 (2006) pp. 385-386.

11. Goers D., Holzapfel M., Scheifele M., Lehmann E., Vontobel P. and P. Novák, J. Power Sources 130 (2004) pp. 221-226.

12. Fischer C. O., Stade J., Bock W., Proc. $5^{\text {th }}$ World Conference on Neutron Radiography. June 17-20, 1996, Berlin, Germany.

13. Lewis W. J., Bennett L. G. I., Chalovick T. R., Francescone O., in: $15^{\text {th }}$ World Conference on Nondestructive Testing, October 15-21, 2000, Roma, Italy. 
14. Stanojev Pereira M. A., Pugliesi F., Pugliesi R., Brazilian Journal of Physics, 38 (3A) (2008) 346.

15. Ferger T., Abele H., Brunner J., Gaehler R., Schillinger B., Villard J. R., The new station for fast radiography and tomography at the ILL in Grenoble Proc. $7^{\text {th }}$ World Conf. on Neutron Radiography (WCNR-7, Rome, Italy, 2005) ed P Chirco and R Rosa (Rome: ENEA).

16. Festa G., Caroppi P. A., Filabozzi A., Andreani C., Arancio M. L., Triolo R., Celso F. Lo., Benfante V., Imberti S., Meas. Sci. Technol. 19 (2008) 034004.

17. Hickner M. A., Siegel N. P., Chen K. S., Hussey D. S., Jacobson D. L., Arif M., J. Electrochem. Soc. 155 (2008) B427.

18. Robinson A. P., Lewin P. L., Sutton S. J., Swingler S. G., "Inspection of high voltage cables using X-ray techniques", Proc. IEEE Symposium on Electrical Insulation, Indianapolis, USA, September 19-22, 2004, pp. 372375.

19. Robinson A. P., Lewin P. L., Sutton S. J., Swingler S. G., "Automated high voltage cable inspection using X-ray images", Proc. IEEE International Conference on Solid Dielectrics, Toulouse, France, July 5-9, 2004.

20. Robinson A. P., Lewin P. L., Swingler S. G., Sutton S. J., Sci. Meas. Technol., 152 (2005) 187.
21. Spowart A. R., Journal of Physics E: Scientific Instruments 5 (1972) 497.

22. MacGillivray G., Imaging with Neutrons: The Other Penetrating Radiation, Nray Services Inc, Proc. SPIE 4142, 48, 2000.

23. Pelowitz D. B., MCNPXTM USER'S MANUAL Version 2.5.0, 2005

24. D'Mellow B., Thomas D. J., Joyce M. J., Kolkowski P., Roberts N. J., Monk S. D., Nucl. Instrum. Meth. Phys. Res. A 577 (2007) 690.

25. NSD-Fusion GmbH, NSD-NG-1e9-DD-P Neutron Generator preliminary description, 2008 (private communication with company).

26. Berger H., Iddings F., Neutron Radiography A state-of-the-Art report, Nondestuctive Testing Information Analysis Center 1998.

27. Hussein E. M. A., Handbook on Radiation Probing, Gauging, Imaging and Analysis Volume II Applications and Design, Kluwer Academic Publishers 2004.

28. Nexans Deutschland Industries, Energy Networks / ESP, 1.9.2006

29. Nexans, Power cables $1-30 \mathrm{kV}$, Edition 2004.

30. Hayashi T., Tobita K., Nishio S., et al., Fus. Eng. Des. 81 (2006) 1285.

31. Hayashi T., Tobita K., Nakamori Y., Orimo S., Journal of Nuclear Materials 386 (2009) 119.

32. Mikerov V., Waschkowski W., Nucl. Instr. and Meth. 424 (1999) 48. 